\title{
Tunable microwave sensor for material testing
}

\author{
Archana Tiwari ${ }^{1}$ and M. Jaleel Akhtar ${ }^{1,2 *}$ \\ ${ }^{1}$ Department of Materials Science Programme, Indian Institute of Technology Kanpur, Kanpur- \\ 208016, India \\ ${ }^{2}$ Department of Electrical Engineering, Indian Institute of Technology Kanpur, Kanpur-208016, India \\ *Email:mjakhtar@iitk.ac.in
}

In this work, an attractive tunable microwave sensor has been proposed for testing the materials as well as the chemicals to be used in chemical industry. This can be used for both sensing and tuning applications. This can also be used for sensing the dispersive materials i.e. materials whose dielectric properties change with frequency.So, for this, the varactor loaded complementary split ring resonator (VLCSRR) is presented and thanks to the presence of varactor diodes, the electrical characteristics of the particle can be electronically tuned. Thus, by loading a microstrip line with VLCSRRs, the structure exhibits a tunable rejection band, which is attributed to the effective permittivity provided by the particle. The VLCSRR based non-invasive sensor is demonstrated using planar technology, which has many advantages such as the low cost, portability. The CSRR is excited using electric field of the microstrip, which is normal to the plane of CSRR. The CSRR is etched in the ground plane of microstrip planar line, which shows characteristics similar to the narrow band stop filter.
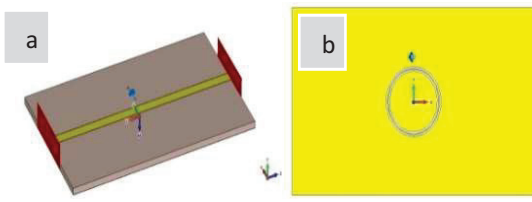

Figure 1: (a) Top view and (b) bottom view of the microstrip line with VLCSRR on the ground plane

Also a microfluidic channel made up of PDMS Polydimethylsiloxane is presented which is placed over CSRR area of sensor. This kind of sensor is suited for biomedical applications.

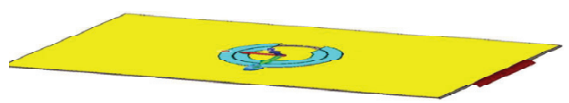

Figure 2: Sensor with PDMS microfluidic channel
The design of the sensor is small electrical resonator i.e. Complementary Split Ring Resonator (CSRR) on $1.32 \mathrm{~mm}$ thick Rogers Substrate. This small resonator is excited by transmission line i.e high impedance line. This resonator has a resonant frequency of $1.25 \mathrm{GHz}$ when simulated on Computer Simulation Technology-Microwave Studios (CST-MWS). It works on the principle of change in the resonant frequency of CSRR when material under test comes in contact with sensor. This shift in the resonant frequency is measured by measuring $\mathrm{S}$ parameter i.e. $\mathrm{S}_{21}$ (insertion loss).

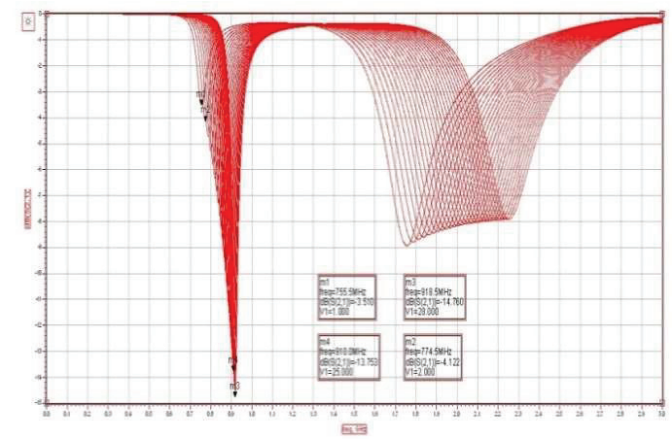

Figure 3: Simulated S-parameter (showing $28 \%$ tuning range) of the microstrip line with VLCSRR on the ground plane

\section{References}

1. I Gil, J. García-García, J. Bonache, F. Martín, M. Sorolla, and R. Marqués, "Varactor-loaded split rings resonators for tuneable notch filters atmicrowave frequencies," Electron. Lett., vol. 40, pp. 1347-1348, Oct. 2004.

2. J. Bonache, M. Gil, I. Gil, J. García-García, and F. Martín "the electrical characteristics of complementary metamaterial resonators,'IEEE Microw. Wireless Compon. Lett., vol. 16, oct 2006. 